\title{
Application of Quadratic Linearization for the Control of Permanent Magnet Synchronous Motor
}

\author{
Parvathy Ayalur Krishnamoorthy, ${ }^{1}$ Kamaraj Vijayarajan, ${ }^{2}$ and Devanathan Rajagopalan ${ }^{1}$ \\ ${ }^{1}$ Hindustan Institute of Technology and Science, Chennai 600 016, India \\ ${ }^{2}$ SSN College of Engineering, Chennai 600 004, India \\ Correspondence should be addressed to Parvathy Ayalur Krishnamoorthy, akparvathy@hindustanuniv.ac.in
}

Received 30 June 2011; Accepted 8 August 2011

Academic Editor: Henry S. H. Chung

Copyright ( 2011 Parvathy Ayalur Krishnamoorthy et al. This is an open access article distributed under the Creative Commons Attribution License, which permits unrestricted use, distribution, and reproduction in any medium, provided the original work is properly cited.

\begin{abstract}
Many of the existing control methods for the permanent magnet synchronous motor (PMSM) either deal with steady state models or consider dynamic models under particular cases. A dynamic model of the PM machine allows powerful control-theoretic techniques such as linearization to be applied to the system. Existing exact feedback linearization of dynamic model of PMSM suffers from singularity issues. In this paper, we propose a quadratic linearization approach for PMSM based on the approximate linearization technique which does not introduce singularities. A MATLAB simulation is used to verify the effectiveness of the linearization technique proposed. Also, to account for higher-order and unmodelled dynamics of PMSM, tuning of the linearizing transformation is proposed and verified using simulation.
\end{abstract}

\section{Introduction}

Permanent magnet (PM) machines, particularly at low power range, are widely used in the industry because of their high efficiency. They have gained popularity in variable frequency drive applications. The merits of the machine are elimination of field copper loss, higher power density, lower rotor inertia, and a robust construction of the rotor [1].

Steady state models, such as in [2], have been used to develop control strategies for the permanent magnet synchronous motor. Vector control [3] enables independent control over the magnitude and angle of the current with respect to the rotor such that instantaneous control over torque is possible. But vector control implemented, in practice [4], assumes a constant flux in the closed loop and uses static models to effect the control scheme. The steady state models used to describe the machine behaviour do not capture explicitly the dynamics of the machine involved. Decoupled control [5], while considering the dynamics of the machine, corresponds to application of particular control strategies for machine control.

A dynamic model of a PM machine using direct and quadrature axis variables [1] allows more powerful general control theories to be brought to bear on the problem of the control of PM machine. One such control-theoretic approach is quadratic linearization of the PM machine model. Linearization of a nonlinear system allows a simple fixed controller to be applied for the control of the linearized system and yet get a uniform closed loop response for different reference and load conditions.

Bodson and Chiasson [6] have designed a controller using differential geometric method based on exact feedback linearization. The main drawback with this method and its variations [7] is that, even if the system is linearizable, the linearizability is subject to certain function of the state being nonsingular in a region of operation of the machine. This puts a constraint on the practical implementation of the control strategy. Zhu et al. [8] provide a static feedback linearization of a PM model (see [8, equation (25)]) which has a simpler quadratic term compared to our model. With the assumption that $L_{d}=L_{q}$, the solution of quadratic linearization becomes trivial. Our method is more general and is applicable to PMSM where $L_{d} \neq L_{q}$.

Krener [9] formulated an approximate feedback linearization technique based on Taylor series expansion; Kang and Krener $[10,11]$ extended the work of Poincare [12] on 
the lines of Krener to consider quadratic linearization of control affine systems. Quadratic linearization can be applied to a PMSM model [1] as the model has a predominant quadratic nonlinearity. But being an approximate technique, higher-order terms are introduced. Consideration of core loss involves fourth-order terms involving product of squares of both current and angular velocity. These together with the stray losses and unmodelled dynamics coupled with the third- and higher-order nonlinearities introduced due to quadratic linearization are best accounted for by tuning the transformations against an actual PM machine on the lines similar to those proposed by Levin and Narendra [13].

In this paper, input and state transformations are derived for a 4-dimensional PM machine model in order to linearize PMSM machine model. The PMSM model is quadratic linearized using the approximate technique. Tuning rules are derived for the linearizing transformations to account for higher-order terms, by back propagation of error between the outputs of quadratic linearized system with a normal form output.

The linearization technique is verified using SIMULINK model which is developed for interior permanent magnet (IPM) machine. The core loss which consists of higher-order terms is included in the SIMULINK model of PMSM, and tuning rules are also simulated. The closed loop response and open loop gain for the system before and after tuning are obtained.

The simulation results after linearization indicate a uniform closed loop response for different reference and load conditions, thus verifying the theory. The simulation results after tuning also verify the effectiveness of tuning.

To summarise the rest of the paper, in Section 2, background required for quadratic linearization is given. In Section 3, the model of PM synchronous motor is derived and is reduced to normal form. In Section 4, PMSM model is linearized. In Section 5, a SIMULINK model of PMSM is constructed and simulation is presented to show the effectiveness of the proposed method. In Section 6, tuning of the transformation coefficients is derived by including core loss in the PMSM model and also simulation results are given to verify the effectiveness of tuning. In Section 7, the paper is concluded.

\section{Background}

Consider a single input control affine system of the form [10]

$$
\begin{aligned}
\dot{x}= & A x+B u+f^{(2)}(x)+f^{(3)}(x)+\cdots+f^{(m)}(x)+\cdots \\
& +g^{(1)}(x)+g^{(2)}(x)+\cdots+g^{(m-1)}(x)+\cdots,
\end{aligned}
$$

where $A$ and $B$ are matrices in the controller normal form

$$
A=\left[\begin{array}{ccccc}
0 & 1 & 0 & \cdots & 0 \\
0 & 0 & 1 & . . & 0 \\
. & . & . & . & . \\
0 & 0 & . . & . . & 1 \\
0 & 0 & 0 & . . & 0
\end{array}\right] ; \quad B=\left[\begin{array}{l}
0 \\
0 \\
\cdot \\
. \\
1
\end{array}\right]
$$

$A$ is an $n \times n$ constant matrix and $B$ is an $n \times 1$ constant matrix.

$x=\left[\begin{array}{llll}x_{1} & x_{2} & \cdots & x_{n}\end{array}\right]^{T}$ and $u$ is a scalar input. $f^{(m)}(x)$, $g^{(m-1)}(x)$ are vector homogeneous polynomials of order $m$ and $(m-1)$, respectively, $m=2,3, \ldots$.

In order to linearize the system, a change of coordinates and feedback $[10,11]$ of the following form is considered:

$$
\begin{gathered}
y=x+\phi(x), \\
u=(1+\beta(x)) v+\alpha(x),
\end{gathered}
$$

where $y=\left[\begin{array}{llll}y_{1} & y_{2} & \cdots & y_{n}\end{array}\right]^{T}, v$ is a scalar, and $\alpha(x), \beta(x)$, and $\phi(x)$ are polynomials given by

$$
\begin{aligned}
& \phi(x)=\phi^{(2)}(x)+\phi^{(3)}(x)+\cdots+\phi^{(m)}(x)+\cdots, \\
& \alpha(x)=\alpha^{(2)}(x)+\alpha^{(3)}(x)+\cdots+\alpha^{(m)}(x)+\cdots, \\
& \beta(x)=\beta^{(1)}(x)+\beta^{(2)}(x)+\cdots+\beta^{(m-1)}(x)+\cdots .
\end{aligned}
$$
to

Applying the transformations (3) and (4), (1) is reduced

$$
\dot{y}=A y+B v
$$

provided that the following equations, called generalized homological equations [10], are satisfied:

$$
\begin{aligned}
& -A \phi^{(m)}(x)+B \alpha^{(m)}(x)+f^{\prime(m)}(x)+\frac{\partial \phi^{(m)}(x)}{\partial x} A x=0, \\
& B \beta^{(m-1)}(x) v+\frac{\partial \phi^{(m)}(x)}{\partial x} B v+g^{\prime(m-1)}(x) v=0 ; \quad \forall v,
\end{aligned}
$$

where $f^{\prime(m)}(x)=f^{(m)}(x)$ for $m=2$ and $f^{\prime(m)}(x)$ is expressed in terms of $f^{(m-i)}(x), i=0,1,2 \ldots(m-2)$ and $\phi^{(m-j)}(x)$, $j=1,2 \ldots(m-2), m>2 \cdot g^{\prime(m-1)}(x)=g^{(1)}(x), m=2$ and $g^{\prime(m-1)}(x)$ is expressed in terms of $g^{(m-i)}(x), i=1,2 \ldots(m-$ $1)$ and $\phi^{(m-j)}(x), j=1,2 \ldots(m-2), m>2$.

Remark 1. Quadratic linearization involves specialization of the above result for $m=2$. Consider

$$
\dot{x}=A x+B u+f^{(2)}(x)+g^{(1)}(x)+O^{(3)}(x, u),
$$

where $A$ and $B$ are in controller normal form [10] and $O^{(3)}(x, u)$ corresponds to terms of order 3 or more. Equations (3) and (4) for $m=2$ can be written as

$$
\begin{gathered}
y=x+\phi^{(2)}(x), \\
u=\left(1+\beta^{(1)}(x)\right) v+\alpha^{(2)}(x) .
\end{gathered}
$$

Applying (9) and (10), (8) can be written as

$$
\dot{y}=A y+B v+O^{\prime(3)}(y, v)
$$

provided that the homological equations

$$
\begin{gathered}
-A \phi^{(2)}(x)+B \alpha^{(2)}(x)+f^{(2)}(x)+\frac{\partial \phi^{(2)}(x)}{\partial x} A x=0, \\
B \beta^{(1)}(x) v+\frac{\partial \phi^{(2)}(x)}{\partial x} B v+g^{(1)}(x) v=0 ; \quad \forall v
\end{gathered}
$$

can be solved for $\phi^{(2)}(x), \alpha^{(2)}(x)$, and $\beta^{(1)}(x)$. 


\section{Machine Model}

The PM machine model can be derived $[1,14,15]$ as

$$
\begin{gathered}
\dot{x}=A x+B u+f^{(2)}(x) \\
x=\left[\begin{array}{llll}
x_{1} & x_{2} & x_{3} & x_{4}
\end{array}\right]^{T}=\left[\begin{array}{llll}
\theta & \omega_{e} & i_{q} & i_{d}
\end{array}\right]^{T}, \\
u=\left[\begin{array}{ll}
u_{1} & u_{2}
\end{array}\right]^{T}=\left[\begin{array}{ll}
v_{q} & v_{d}
\end{array}\right]^{T},
\end{gathered}
$$

where $v_{q}, v_{d}, i_{q}$, and $i_{d}$ represent the quadrature and direct axis voltages and currents, respectively, and $\theta$ and $\omega_{e}$ represent rotor position and angular velocity, respectively.

$$
\begin{gathered}
A=\left[\begin{array}{cccc}
0 & 1 & 0 & 0 \\
0 & 0 & \frac{1.5 p \lambda}{J} & 0 \\
0 & \frac{-\lambda p}{L_{q}} & \frac{-R}{L_{q}} & 0 \\
0 & 0 & 0 & \frac{-R}{L_{d}}
\end{array}\right], \\
\left.B=\left[\begin{array}{cc}
0 & 0 \\
0 & 0 \\
\frac{1}{L_{q}} & 0 \\
0 & \frac{1}{L_{d}}
\end{array}\right], \quad \begin{array}{c}
\frac{1.5 p\left(L_{d}-L_{q}\right) i_{d} i_{q}}{J} \\
\frac{-L_{d} p \omega_{e} i_{d}}{L_{q}} \\
\frac{L_{q} p \omega_{e} i_{q}}{L_{d}}
\end{array}\right],
\end{gathered}
$$

$\lambda$ is the flux induced by the permanent magnet of the rotor in the stator phases. $L_{d}$ and $L_{q}$ are the direct and quadrature inductances, respectively. $R$ is the stator resistance, $p$ is the number of pole pairs, and $J$ is the system moment of inertia.

The model (13) can be reduced, in a standard way using linear change of coordinates and feedback [16], to the Brunovsky form [17] for two inputs as in (16) below (where $x, u, A$, and $B$ are retained for simplicity of notation):

$$
\dot{x}=A x+B u+f^{(2)}(x),
$$

where

$$
\begin{gathered}
A=\left[\begin{array}{llll}
0 & 1 & 0 & 0 \\
0 & 0 & 1 & 0 \\
0 & 0 & 0 & 0 \\
0 & 0 & 0 & 0
\end{array}\right], \quad B=\left[\begin{array}{ll}
0 & 0 \\
0 & 0 \\
1 & 0 \\
0 & 1
\end{array}\right], \quad f^{(2)}(x)=\left[\begin{array}{c}
0 \\
k_{1} x_{3} x_{4} \\
k_{2} x_{2} x_{4} \\
k_{3} x_{2} x_{3}
\end{array}\right], \\
k_{1}=\frac{1.5 p\left(L_{d}-L_{q}\right) a_{4}}{J a_{1}}, \quad k_{2}=\frac{-L_{d} p a_{1} a_{4}}{L_{q}}, \\
a_{1}=\frac{1.5 p \lambda}{J}, \quad k_{3}=\frac{L_{q} C_{1}^{2} a_{1}}{L_{d} a_{4}}, \quad a_{4}=\frac{-R}{L_{d}}, \quad C_{1}=\frac{1}{L_{q}} .
\end{gathered}
$$

\section{Linearization of Interior Permanent Magnet (IPM) Synchronous Motor}

The quadratic linearization given in Section 2 is extended to the case of two inputs in a straightforward way and applied to a PM machine model in this section $[14,15]$.

Theorem 2. Given the 4-dimensional model of a PM synchronous motor (IPM model) of the form (16), the system can be linearized using the following transformations:

$$
y=x+\phi^{(2)}(x)
$$

$$
u=\left(I_{2}+\beta^{(1)}(x)\right) v+\alpha^{(2)}(x)
$$

where $u=\left[\begin{array}{ll}u_{1} & u_{2}\end{array}\right]^{T}, v=\left[\begin{array}{ll}v_{1} & v_{2}\end{array}\right]^{T}$,

$$
\begin{gathered}
\phi^{(2)}(x)=\left[\begin{array}{c}
0 \\
0 \\
k_{1} x_{3} x_{4} \\
0
\end{array}\right], \\
\alpha^{(2)}(x)=\left[\begin{array}{c}
-k_{2} x_{2} x_{4} \\
-k_{3} x_{2} x_{3}
\end{array}\right],
\end{gathered}
$$

$$
\beta^{(1)}(x)=(-1)\left(B^{T} \frac{\partial \phi^{(2)}(x)}{\partial x} B\right)=-\left[\begin{array}{cc}
k_{1} x_{4} & k_{1} x_{3} \\
0 & 0
\end{array}\right],
$$

where $I_{2}$ is the identity matrix of order 2.

The system then reduces to

$$
\dot{y}=A y+B v+O^{(3)}(y, v)
$$

where $O^{(3)}(y, v)$ represents third-and higher-order nonlinearities.

Proof. Applying transformations (18) and (19) (which correspond to a natural extension of (9) and (10) to two inputs) to (16), the homological equations to be considered can be written as

$$
\begin{gathered}
-A \phi^{(2)}(x)+B \alpha^{(2)}(x)+f^{(2)}(x)+\frac{\partial \phi^{(2)}(x)}{\partial x} A x=0, \\
B \beta^{(1)}(x)+\frac{\partial \phi^{(2)}(x)}{\partial x} B=0 .
\end{gathered}
$$




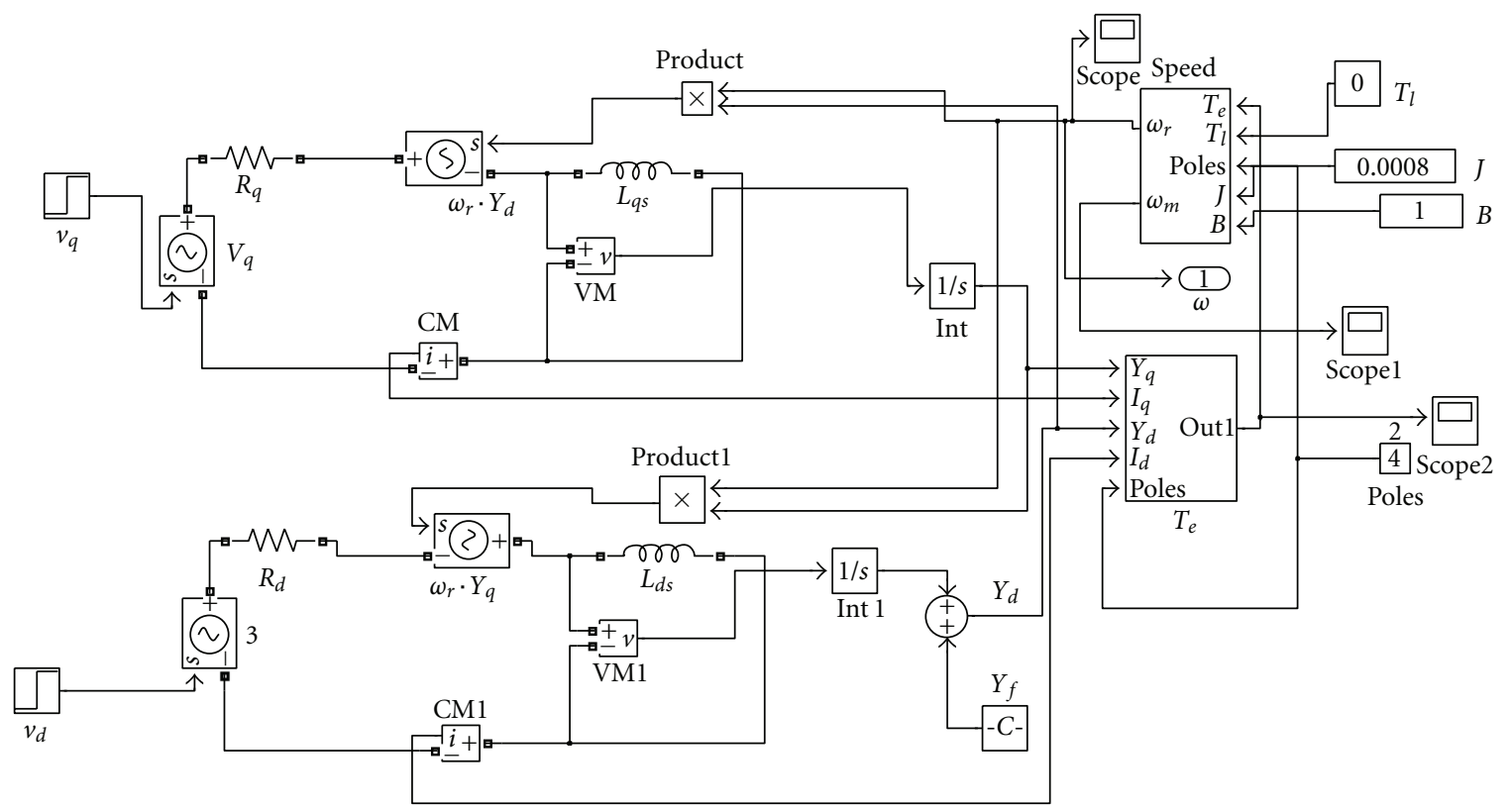

FIgURE 1: PMSM design using SIMULINK.

By choosing $\phi^{(2)}(x)$ and $\alpha^{(2)}(x)$ as in (20) and (21), (24) reduces to

$$
\begin{gathered}
{\left[\begin{array}{c}
0 \\
k_{1} x_{3} x_{4} \\
0 \\
0
\end{array}\right]+\left[\begin{array}{c}
0 \\
0 \\
-k_{2} x_{2} x_{4} \\
-k_{3} x_{2} x_{3}
\end{array}\right]+\left[\begin{array}{c}
0 \\
k_{1} x_{3} x_{4} \\
k_{2} x_{2} x_{4} \\
k_{3} x_{2} x_{3}
\end{array}\right]} \\
+\left[\begin{array}{cccc}
0 & 0 & 0 & 0 \\
0 & 0 & 0 & 0 \\
0 & 0 & k_{1} x_{4} & k_{1} x_{3} \\
0 & 0 & 0 & 0
\end{array}\right]\left[\begin{array}{c}
x_{2} \\
x_{3} \\
0 \\
0
\end{array}\right]=0 .
\end{gathered}
$$

Premultiplying (25) by $B^{T}$ and noting that $B^{T} B=I_{2},(25)$ reduces to

$$
\beta^{(1)}(x)=(-1)\left(B^{t} \frac{\partial \phi^{(2)}(x)}{\partial x} B\right)
$$

Also, substitution of (27) satisfies (25). Hence, the homological equations are satisfied and quadratic linearization is achieved, hence the proof.

\section{Experimental Simulation Results}

Given the parameters $R=2.875 \Omega, L_{q}=9 \mathrm{mH}, L_{d}=$ $7 \mathrm{mH}, \omega_{e}=3500 \mathrm{rpm}, p=4, J=0.0008 \mathrm{kgm}^{2}$ and $\lambda=$ $175 \mathrm{mWb}$ turns, of an actual PM machine, Figure 1 shows the SIMULINK model of the PMSM which is constructedby using speed and torque blocks and control circuit. $v_{q}$ and $v_{d}$ are taken as inputs to the motor. The model is reduced to normal form in a standard way by using the linear transformations. The PM model in Figure 1 is especially
TABLE 1: Steady state gain of $\omega_{e}$ versus $v_{q}$ for the system in open loop (prior to linearization).

\begin{tabular}{lcc}
\hline$v_{q}$ & $\omega_{e}$ & $k=d \omega_{e} / d v_{q}$ \\
\hline 5 & 2.55 & - \\
10 & 4.7709 & 0.44418 \\
15 & 6.4706 & 0.33994 \\
20 & 7.6082 & 0.22752 \\
25 & 8.2567 & 0.1297 \\
30 & 8.5326 & 0.05518 \\
\hline
\end{tabular}

configured for the IPM where $L_{d} \neq L_{q} . N_{1}$ and $N_{2}$ blocks in Figure 2 include the above linear transformation and the nonlinear transformation (18) and (19) as well.

Prior to linearization, the open loop steady state gain of $\omega_{e}$ versus $v_{q}$ of the PMSM model is investigated and the results are given in Table 1 . In this table, it is observed that the open loop steady state gain of $\omega_{e}$ versus $v_{q}$ (keeping $v_{d}$ constant) is not constant because of the system nonlinearity. To verify the linearity of the system after linearization, we investigated the variation of its gain of $y_{2}$ (a scaled version of $\omega_{e}$ as can be seen from (18) and (20)) with input $v_{1}$ (see Figure 2) and the results are given in Table 2. The table reveals that the gain of the system is nearly constant, thus verifying that by applying the homogeneous linearizing transformation, the PMSM model is made nearly linear for the given set of inputs.

We now proceed to show that the nearly constant gain of the linearized model results in a uniform closed loop response on a range of set point and load inputs with a fixed controller. This is in contrast to the case before linearization under the corresponding conditions. 


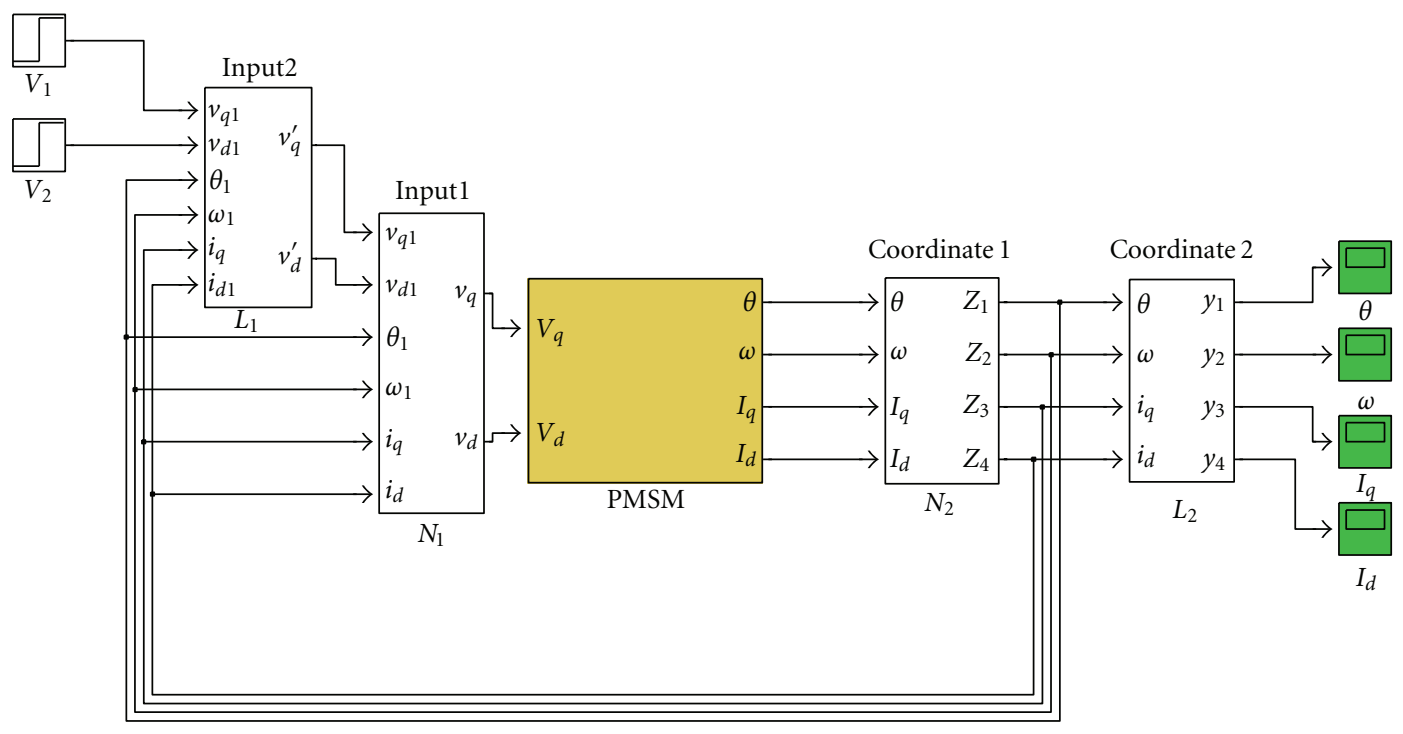

FIgURe 2: Linearization of PMSM.

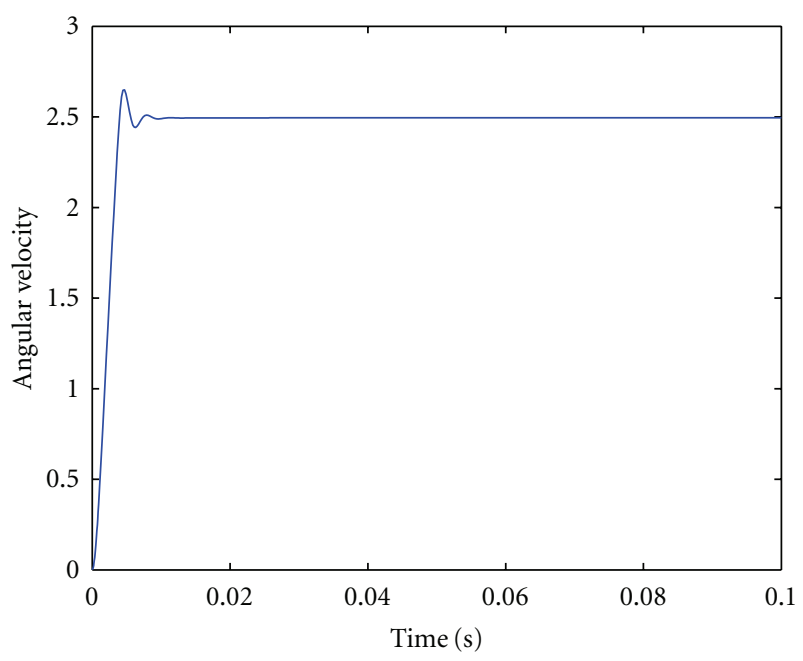

FIgURE 3: Time response of angular velocity in closed loop when $v_{q}=5 ; k_{p}=50 ; k_{i}=2$ (before linearization).

TABLE 2: Steady state gain of $y_{2}$ versus $v_{1}$ for the linearized system in open loop.

\begin{tabular}{lcc}
\hline$v_{1}$ & $\begin{array}{c}y_{2} \\
\times 10^{-6}\end{array}$ & $\begin{array}{c}k=d y_{2} / d v_{1} \\
\times 10^{-6}\end{array}$ \\
\hline 5 & 2.176 & - \\
10 & 4.366 & 0.438 \\
15 & 6.585 & 0.4438 \\
20 & 8.845 & 0.452 \\
25 & 11.162 & 0.4634 \\
30 & 13.55 & 0.4776 \\
\hline
\end{tabular}

Figures 3 and 4 show the time response of angular velocity $\omega_{e}$ by closing the loop around PMSM model before linearization when $v_{q}=5$ and 30 units, respectively. It is

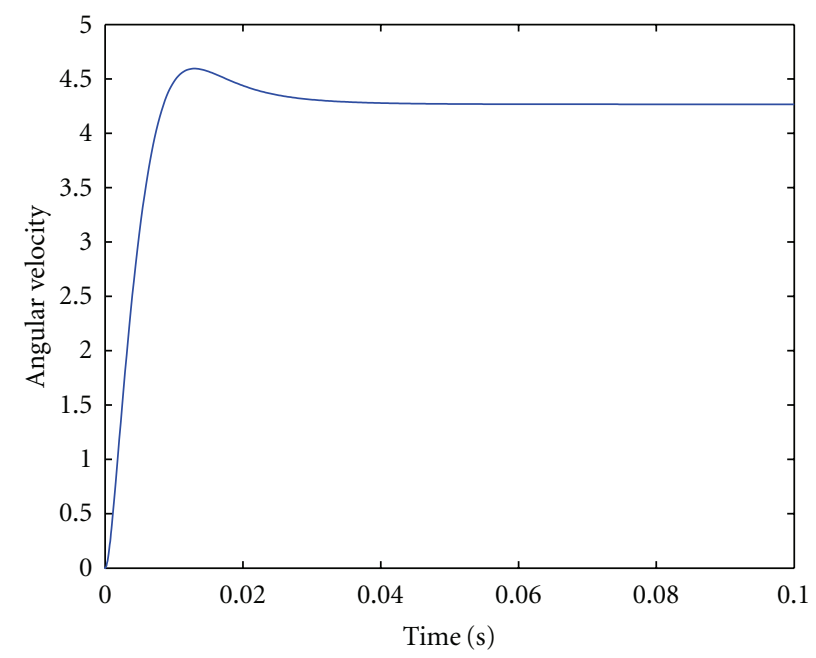

FIGURE 4: Time response of angular velocity in closed loop when $v_{q}=30 ; k_{p}=50 ; k_{i}=2$ (before linearization).

observed that the dynamic response for $v_{q}=5$ is more oscillatory compared to the case of $v_{q}=30$ with a fixed controller of proportional gain $=50$ and integral constant $=$ 2. This is to be expected since the loop gain is higher in the former case with a higher static gain in the motor as can be seen from Table 1.

Figures 5 and 6 show the time response of $y_{2}$ of the transformed PMSM system (Figure 2) in closed loop when $v_{1}=5$ and 30 units, respectively. It is observed that a uniform output response is obtained in the closed loop after linearization when the reference is varied. Since the static gain in Table 2 is nearly uniform, the loop gain is also nearly constant for the extreme points in the operating range, thus resulting in the uniform dynamic responses as in Figures 5 and 6.

Figures 7 and 8 show the time response of angular velocity $\omega_{e}$ of the PMSM model before linearization in closed 


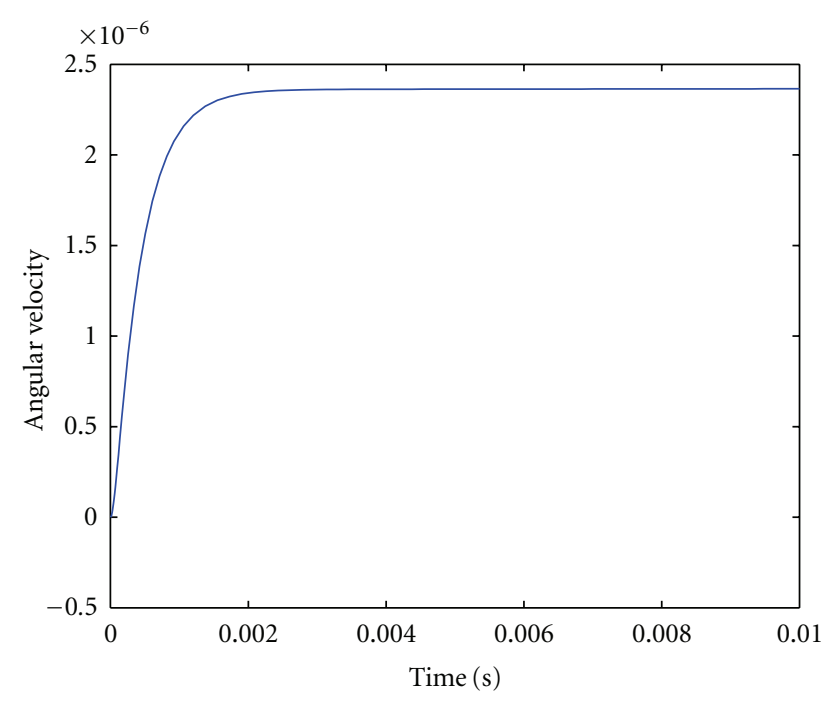

FIgURE 5: Time Response of $y_{2}$ for the linearized system in closed loop when $v_{1}=5 ; k_{p}=50 ; k_{i}=2$.

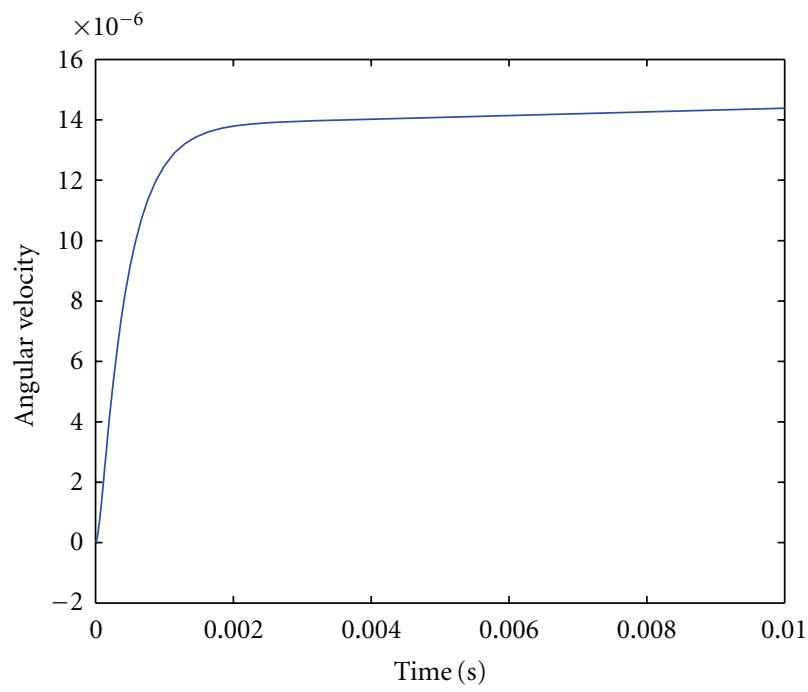

FIGURE 6: Time Response of $y_{2}$ for the linearized system in closed loop when $v_{1}=30 ; k_{p}=50 ; k_{i}=2$.

loop when $v_{q}=15$ units with the load torque $T$ varied as $T=0$ and 1 unit, respectively. Note that the dynamic responses in Figures 7 and 8 are different, with the response of Figure 8 corresponding to an inverse response (i.e., speed reduces first before increasing). The latter corresponds to a response of a nonminimum phase system.

Figures 9 and 10 show the time response of $y_{2}$ of the transformed PMSM system in closed loop when $v_{1}=15$ units and $T=0$ and 1 unit, respectively. It is observed that a uniform output response is obtained in the closed loop after linearization when load torque is varied. Hence, it is verified that a linearized system gives a uniform closed loop response for the different reference and load conditions used for testing as above.

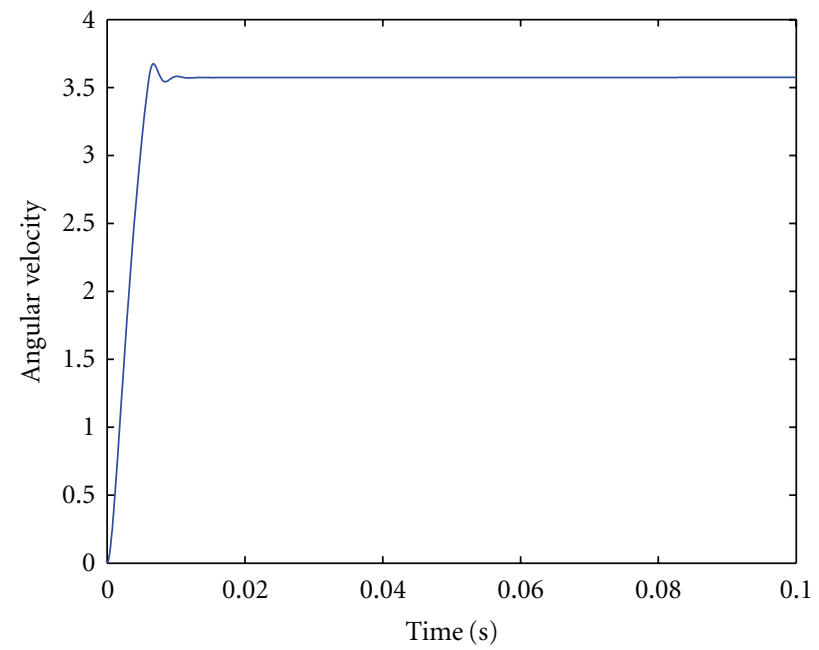

FIgURE 7: Time response of angular velocity in closed loop when $v_{q}=15$ units, $T=0 \mathrm{~N}-\mathrm{m} ; k_{p}=50 ; k_{i}=2$ (before linearizaton).

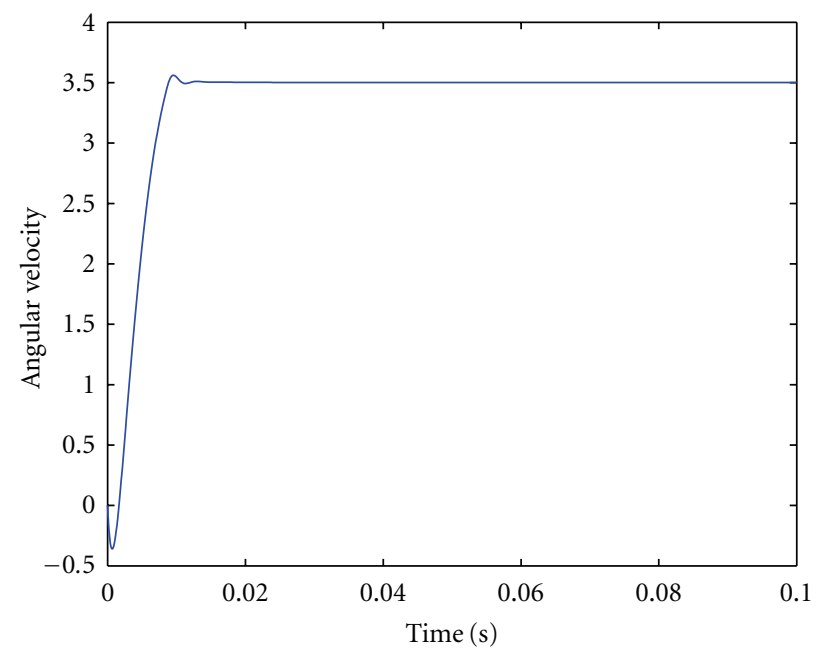

Figure 8: Time response of angular velocity in closed loop when $v_{q}=15$ units, $T=1 \mathrm{~N}-\mathrm{m} ; k_{p}=50 ; k_{i}=2$ (before linearization).

\section{Tuning}

6.1. Core Loss. The core loss or iron loss, caused by the permanent magnet (PM) flux and armature reaction flux, is a significant component in the total loss of a PMSM, and, thus, it can have a considerable effect on the PMSM modeling and performance prediction.

The net core loss $P_{l c}$ [2] for the machine is computed as follows:

$$
P_{l c}=\left[\frac{1.5 \omega_{r}{ }^{2}\left(L_{q} I_{q}\right)^{2}}{R_{c}}+\frac{1.5 \omega_{r}{ }^{2}\left(\lambda_{a f}+L_{d} I_{d}\right)^{2}}{R_{c}}\right],
$$

where $R_{c}$ represents core loss resistance, $\lambda_{a f}$ represents magnet flux linkage and $\omega_{r}$ denotes rotor electrical speed. 


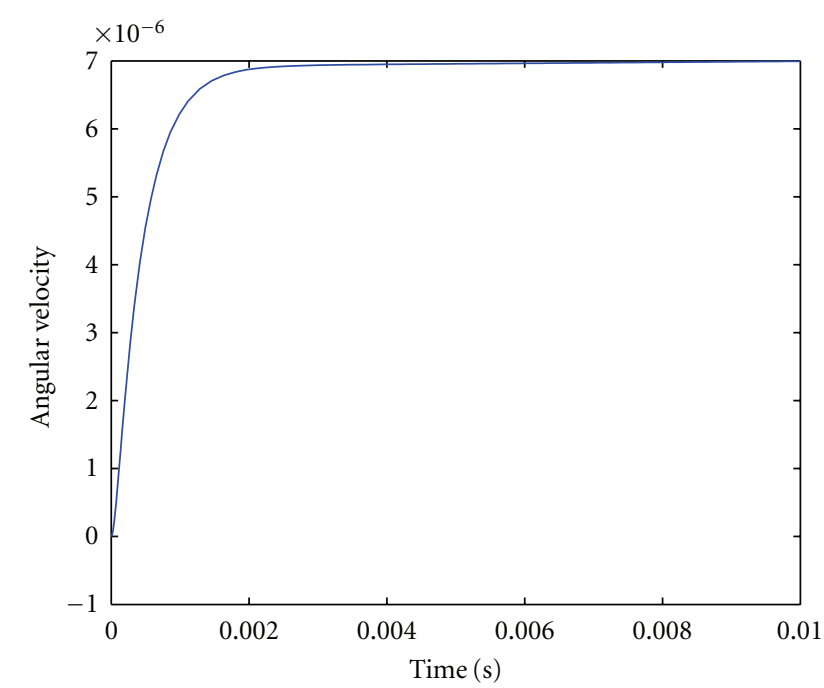

FIGURE 9: Time Response of $y_{2}$ for the linearized system in closed loop when $v_{1}=15$ units, $T=0 \mathrm{~N}-\mathrm{m} ; k_{p}=50 ; k_{i}=2$.

The mechanical torque equation including core losses is given by

$$
\begin{gathered}
T_{e}=\left(\frac{2}{P}\right) J \frac{d \omega_{e}}{d t}+T_{l}+T_{l c}, \\
T_{l c}=\frac{P_{l c}}{\omega_{r}} .
\end{gathered}
$$

6.2. Tuning Formula. To account for the core loss (28), unmodelled dynamics, and higher-order nonlinearities, tuning of the quadratic linearization transformations $N_{1}$ and $\mathrm{N}_{2}$ can be done [13]. Figure 11 shows the block diagram for tuning.

Error $(E)$ can be calculated as

$$
E=\left(\varepsilon^{T} \varepsilon\right)=\left[(y-\hat{y})^{T}(y-\hat{y})\right]^{1 / 2},
$$

where $\varepsilon=\left(\begin{array}{llll}\varepsilon_{1} & \varepsilon_{2} & \varepsilon_{3} & \varepsilon_{4}\end{array}\right)^{T}$.

The error can be written as

$$
E=\left(\varepsilon_{1}^{2}+\varepsilon_{2}^{2}+\varepsilon_{3}^{2}+\varepsilon_{4}^{2}\right)^{1 / 2} .
$$

Since $\phi^{(2)}(x)$ and $\beta^{(1)}(x)$ are both functions of $k_{1}$, we shall redefine

$$
\begin{gathered}
\phi^{(2)}(x)=\left[\begin{array}{c}
0 \\
0 \\
k_{1} x_{3} x_{4} \\
0
\end{array}\right], \\
\beta^{(1)}(x)=-\left[\begin{array}{cc}
k_{1}^{\prime} x_{4} & k_{1}^{\prime} x_{3} \\
0 & 0
\end{array}\right]
\end{gathered}
$$

so that $\phi^{(2)}(x)$ and $\beta^{(1)}(x)$ can be independently tuned by tuning $k_{1}$ and $k_{1}^{\prime}$, respectively. $\alpha^{(2)}(x)$ is not varied.

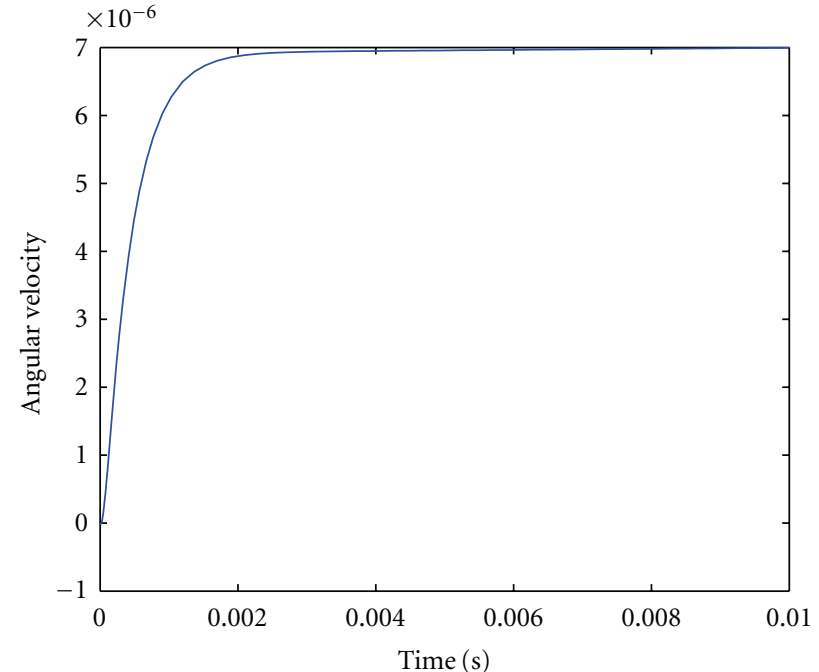

FIGURE 10: Time Response of $y_{2}$ for the linearized system in closed loop when $v_{1}=15$ units, $T=1 \mathrm{~N}-\mathrm{m} ; k_{p}=50 ; k_{i}=2$.

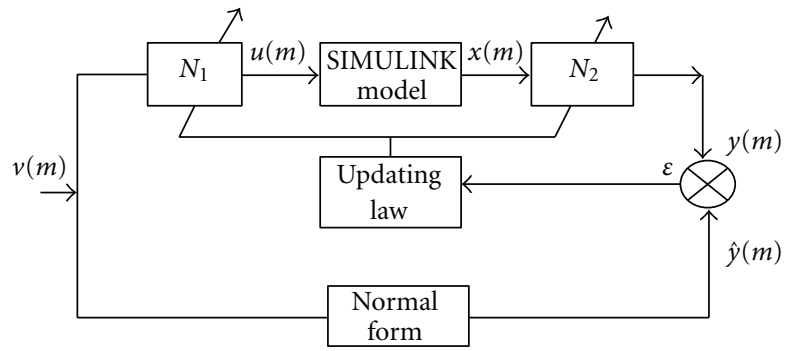

FIGURE 11: Block diagram for tuning of transformation.

6.2.1. Updation of $\mathrm{N}_{2}$ Transformation Coefficients. Tuning of $\mathrm{N}_{2}$ transformation implies the tuning of $\phi^{(2)}(x)$. As $\phi^{(2)}(x)$ is a function of only $k_{1} x_{3} x_{4}$, the coefficient $k_{1}$ has to be updated based on the error between the outputs of quadratic linearized system and normal form. The updation law is derived as follows:

$$
\Delta k_{1}=\frac{\partial E}{\partial k_{1}}=\frac{\partial E}{\partial y} \frac{\partial y}{\partial k_{1}} .
$$

From (31), it is seen that $\partial E / \partial y_{i}=\varepsilon_{i} / E, i=1,2,3,4$.

Hence, $\partial E / \partial y=\left[\begin{array}{llll}\varepsilon_{1} / E & \varepsilon_{2} / E & \varepsilon_{3} / E & \varepsilon_{4} / E\end{array}\right]$,

$$
\therefore \Delta k_{1}=\left[\begin{array}{llll}
\frac{\varepsilon_{1}}{E} & \frac{\varepsilon_{2}}{E} & \frac{\varepsilon_{3}}{E} & \frac{\varepsilon_{4}}{E}
\end{array}\right]\left[\begin{array}{c}
0 \\
0 \\
x_{3} x_{4} \\
0
\end{array}\right]=\frac{\varepsilon_{3}}{E} x_{3} x_{4} .
$$

Updation of $\phi^{(2)}(x)$ is done by using the formula

$$
k_{1}(m)=k_{1}(m-1)-\rho_{1} \Delta k_{1}(m) ; \quad 0<\rho_{1}<1,
$$

where $m$ corresponds to the updating step and $\rho_{1}$ corresponds to the accelerating factor. 


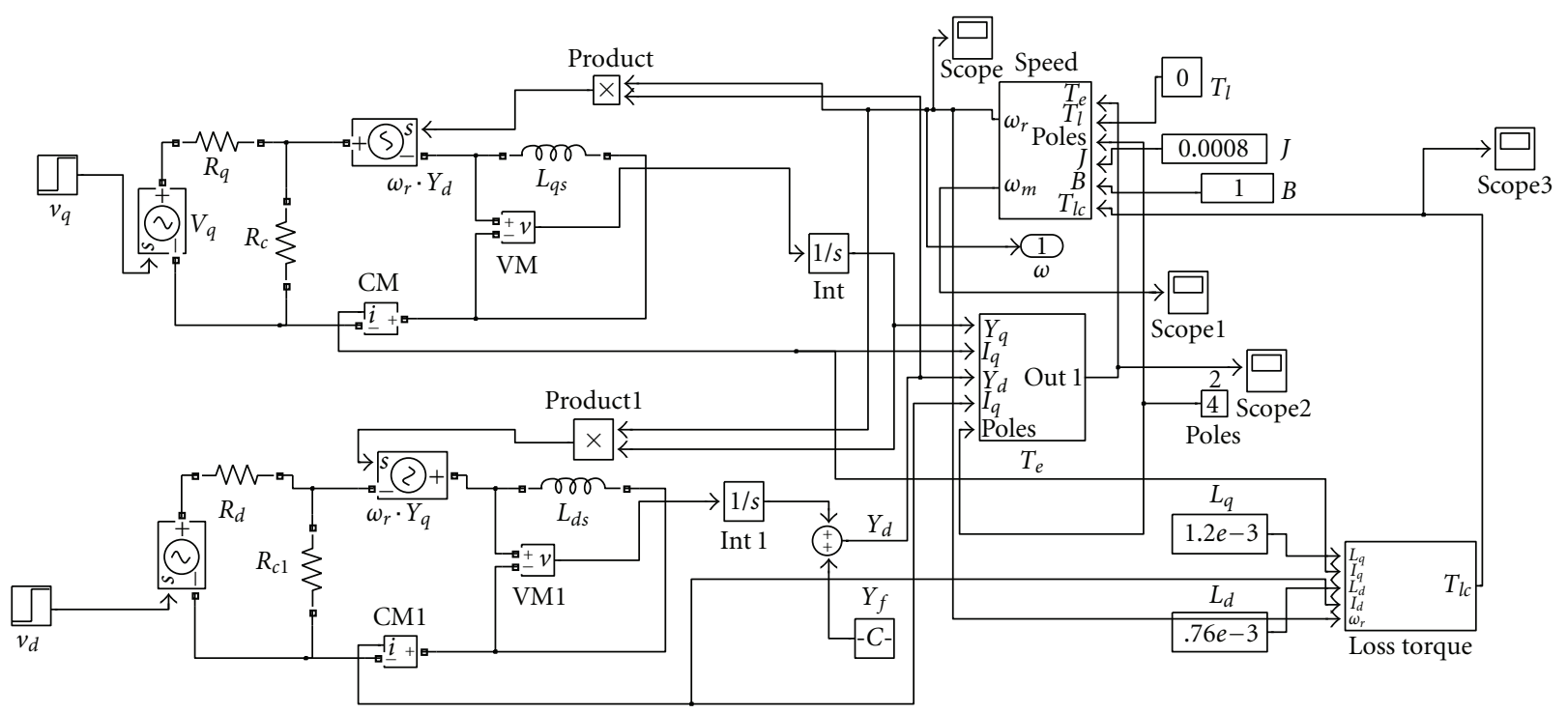

FIGURE 12: PMSM model including core loss.

6.2.2. Updation of $N_{1}$ Transformation Coefficients. Tuning of $N_{1}$ transformation is achieved by tuning of $\beta^{(1)}(x)$. As $\beta^{(1)}(x)$ is a function of $k_{1}^{\prime} x_{3}$ and $k_{1}^{\prime} x_{4}$, the coefficient $k_{1}^{\prime}$ has to be updated based on the error between the outputs of quadratic linearized system and normal form. The updation law is derived as follows:

$$
\Delta k_{1}^{\prime}=\frac{\partial E}{\partial k_{1}^{\prime}}=\frac{\partial E}{\partial y} \frac{\partial y}{\partial x} \frac{\partial x}{\partial u_{1}} \frac{\partial u_{1}}{\partial k_{1}^{\prime}},
$$

where

$$
\begin{gathered}
\frac{\partial E}{\partial y}=\left[\begin{array}{llll}
\frac{\varepsilon_{1}}{E} & \frac{\varepsilon_{2}}{E} & \frac{\varepsilon_{3}}{E} & \frac{\varepsilon_{4}}{E}
\end{array}\right], \\
\frac{\partial y}{\partial x}=\left[\begin{array}{cccc}
1 & 0 & 0 & 0 \\
0 & 1 & 0 & 0 \\
0 & 0 & \left(1+k_{1}^{\prime} x_{4}\right) & k_{1}^{\prime} x_{3} \\
0 & 0 & 0 & 1
\end{array}\right], \quad \frac{\partial x}{\partial u_{1}}=\left[\begin{array}{c}
0 \\
\frac{-1}{k_{2} x_{4}} \\
0 \\
\frac{-1}{k_{2} x_{2}}
\end{array}\right],
\end{gathered}
$$

assuming that the steady state of the SIMULINK model is reached within the tuning period:

$$
\frac{\partial u_{1}}{\partial k_{1}^{\prime}}=-v_{1} x_{4}-v_{2} x_{3}
$$

Thus, $\Delta k_{1}^{\prime}=\left(\left(v_{1} x_{4}+v_{2} x_{3}\right) / E\right)\left(\varepsilon_{2} / k_{2} x_{4}+\left(\varepsilon_{3} k_{1}^{\prime} x_{3}+\varepsilon_{4}\right) / k_{2} x_{2}\right)$. Updation of $\beta^{(1)}(x)$ is done by using the formula

$$
k_{1}^{\prime}(m)=k_{1}^{\prime}(m-1)-\rho_{2} \Delta k_{1}^{\prime}(m), \quad 0<\rho_{2}<1,
$$

where $m$ corresponds to the updating step and $\rho_{2}$ corresponds to the accelerating factor.

6.3. Tuning Simulation Results. PMSM model including core loss is given in Figure 12 where the block due to loss torque
(30) is also included. The tuning of the transformations is done using the updations for $\phi^{(2)}(x)$ and $\beta^{(1)}(x)$ as per formulae (36) and (40) as given in Figure 13. The simulation diagram for tuning is done by using memory blocks to store the updated values of $\phi^{(2)}(x)$ and $\beta^{(1)}(x)$. It is seen that the error after tuning is reduced to 0.01 . Table 3 shows the variation of $y_{2}$ versus $v_{1}$ of the PMSM model after linearization including core loss prior to tuning of the coefficients. It is observed that the open loop steady state gain is not constant due to the effect of core loss. Table 4 shows the variation of $y_{2}$ versus $v_{1}$ for the linearized system after incorporating tuning of the transformation coefficients. The table reveals that the gain of the system is nearly constant thus verifying the effectiveness of tuning. Figures 14 and 15 show the closed loop time response of $y_{2}$ of the linearized system including core loss before tuning when $v_{1}=5$ units and 30 units, respectively; $k_{p}=50 ; k_{i}=2$. Figures 16 and 17 show the closed loop time response of $y_{2}$ of the linearized system including core loss after tuning when $v_{1}=5$ units and 30 units, respectively; $k_{p}=50 ; k_{i}=2$. It is observed that the dynamic response before tuning for $v_{1}=5$ units is more oscillatory compared to the case of $v_{1}=30$ units. This is to be expected since the loop gain is higher in the former case with a higher static gain in the plant or motor as can be seen from Table 3 . Since the static gain in Table 4 is nearly uniform, the loop gain is also nearly constant for the extreme points in the operating range, thus resulting in the uniform dynamic responses in Figures 16 and 17. Thus, the effectiveness of tuning the linearizing transformations to overcome the unmodelled core loss is verified.

\section{Conclusion}

As the PMSM is inherently nonlinear, to design a controller that can provide a predictable uniform performance of the drive under varying operating conditions, it is necessary to 


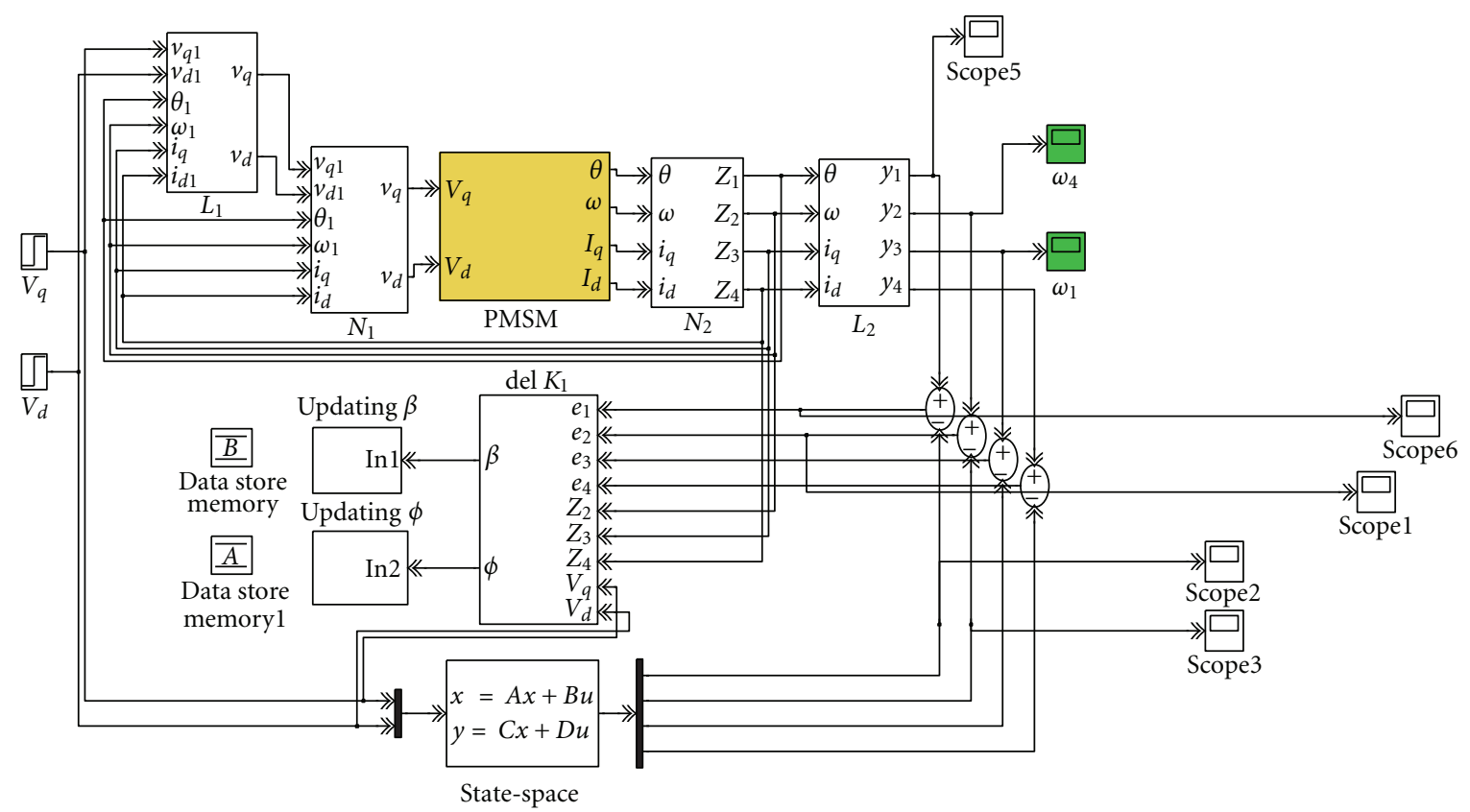

FIgURE 13: Simulation diagram for controller tuning.

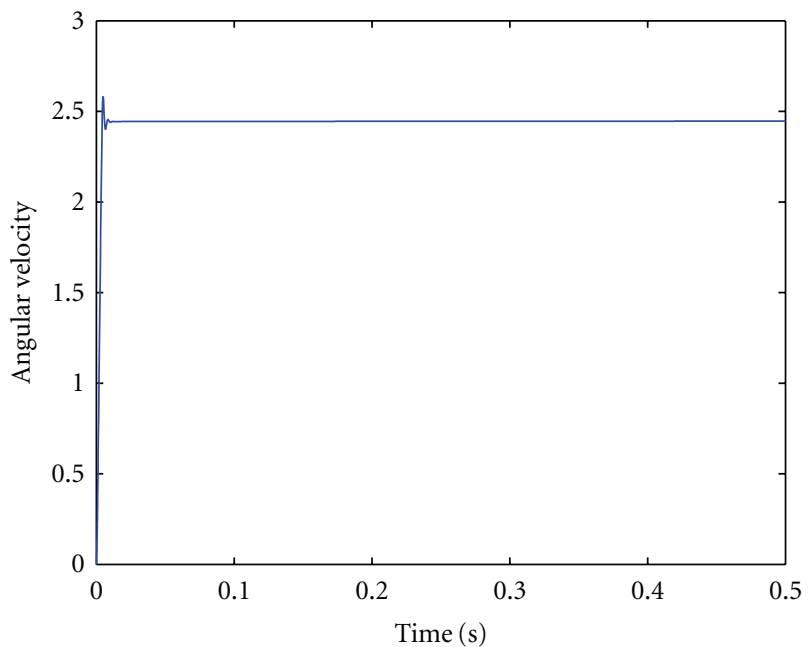

FIGURE 14: Time response of angular velocity in closed loop when $v_{q}=5$ unit; $k_{p}=50 ; k_{i}=2$ before tuning.

TABLE 3: Steady state gain of $y_{2}$ versus $v_{1}$ for the linearized system in open loop before tuning.

\begin{tabular}{lcc}
\hline$v_{1}$ & $\begin{array}{c}y_{2} \\
\times 10^{-6}\end{array}$ & $\begin{array}{c}k=d y_{2} / d v_{1} \\
\times 10^{-6}\end{array}$ \\
\hline 5 & 2.5317 & - \\
10 & 4.673 & 0.4286 \\
15 & 6.2126 & 0.30792 \\
20 & 7.147 & 0.18688 \\
25 & 7.594 & 0.0894 \\
30 & 7.7001 & 0.02122 \\
\hline
\end{tabular}

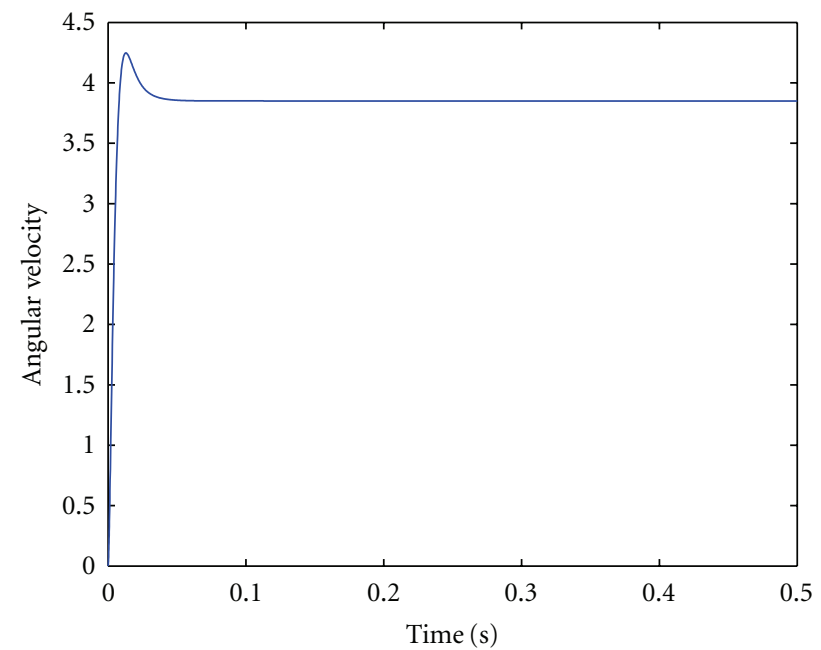

FIGURE 15: Time response of angular velocity in closed loop when $v_{q}=30$ unit; $k_{p}=50 ; k_{i}=2$ before tuning.

TABLE 4: Steady state gain of $y_{2}$ versus $v_{1}$ for the linearized system in open loop after tuning.

\begin{tabular}{lcc}
\hline$v_{1}$ & $\begin{array}{c}y_{2} \\
\times 10^{-6}\end{array}$ & $\begin{array}{c}k=d y_{2} / d v_{1} \\
\times 10^{-6}\end{array}$ \\
\hline 5 & 2.0033 & - \\
10 & 4.0264 & 0.40462 \\
15 & 6.0925 & 0.41322 \\
20 & 8.193 & 0.4201 \\
25 & 10.295 & 0.4204 \\
30 & 12.434 & 0.4278
\end{tabular}




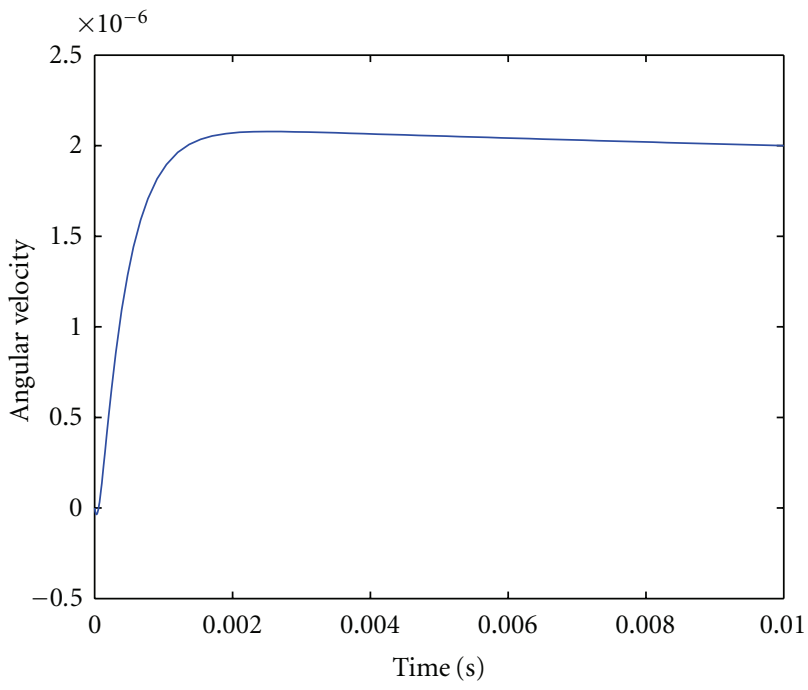

FIgURE 16: Time response of $y_{2}$ for the linearized system in closed loop when $v_{q}=5$ unit; $k_{p}=50 ; k_{i}=2$ after tuning.

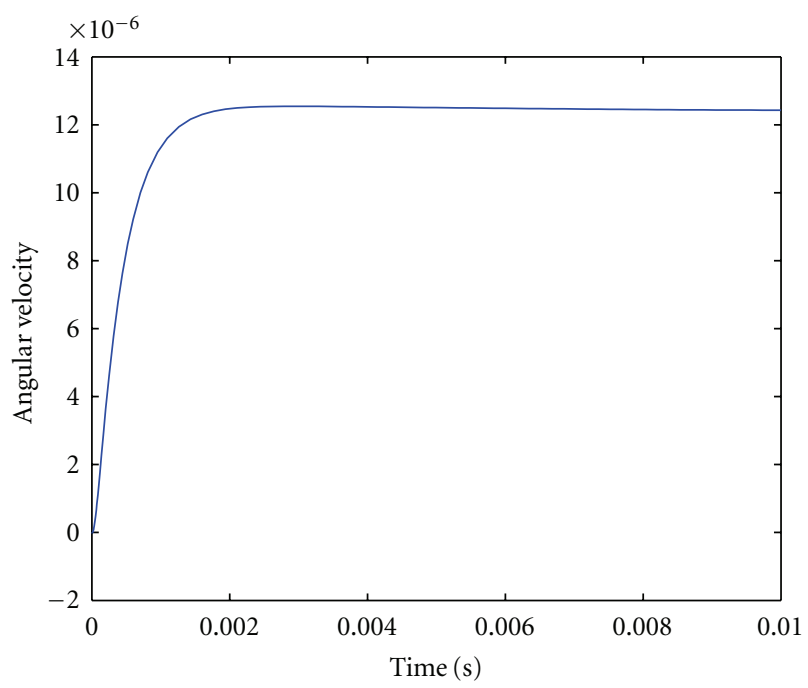

FIgURE 17: Time response of $y_{2}$ for the linearized system in closed loop when $v_{q}=30$ unit; $k_{p}=50 ; k_{i}=2$ after tuning.

linearize the PMSM. In this paper, the dynamic model of a $\mathrm{PM}$ synchronous motor involving quadratic nonlinearity is linearized. The technique used is based on the control input extension of Poincare's work due to Kang and Krener which is in line with the approximate linearization technique of Krener $[9,18]$. The existing techniques of exact feedback linearization introduce singularities in the system, which may cause difficulties in the implementation of the closed loop control. In the proposed method, the problem of singularities does not arise.

The PMSM machine model, together with the state and input transformations, are simulated using SIMULINK. The simulation results show that the quadratic linearizing transformations effectively linearize the system thus, supporting the theory. The simulation verifies that a uniform response under a fixed controller is obtained for the linearized system for variations of reference speed and load conditions, in contrast to the case before linearization.

Further, to account for the core loss, unmodelled dynamics and third- and higher-order nonlinearities, tuning of the transformation parameters is proposed by comparing the output of the linearized system with a normal form output. After tuning, it is shown that the linearized system shows improved linearity in terms of static gain compared to the condition before tuning. Closed loop system response for the linearized system is also shown to be uniform due to the effect of tuning of linearization transformations, under varying reference inputs.

The proposed linearization method can be extended to induction motor and wound synchronous motor models as well [19].

\section{References}

[1] B. K. Bose, Modern Power Electronics and AC Drives, Pearson Education, 2002.

[2] R. Monajemy, Control strategies and parameter compensation of permanent magnet synchronous motor drives, Ph.D. thesis, Virginia Polytechnic Institute and State University, Blachsberg, Va, USA, 2000.

[3] J. K. Seok, J. K. Lee, and D. C. Lee, "Sensorless speed control of nonsalient permanent-magnet synchronous motor using rotor-position-tracking PI controller," IEEE Transactions on Industrial Electronics, vol. 53, no. 2, pp. 399-405, 2006.

[4] P. Pillay and R. Krishnan, "Modeling, simulation, and analysis of permanent-magnet motor drives. I. The permanentmagnet synchronous motor drive," IEEE Transactions on Industry Applications, vol. 25, no. 2, pp. 265-273, 1989.

[5] H. Zhu, X. Xiao, and Y. Li, "PI type dynamic decoupling control scheme for PMSM high speed operation," in Proceedings of the 25th Annual IEEE Applied Power Electronics Conference and Exposition (APEC '10), pp. 1736-1739, Palm Springs, Calif, USA, February 2010.

[6] M. Bodson and J. Chiasson, "Differential-geometric methods for control of electric motors," International Journal of Robust and Nonlinear Control, vol. 8, no. 11, pp. 923-954, 1998.

[7] J. Chiasson, "Nonlinear controllers for an induction motor," Control Engineering Practice, vol. 4, no. 7, pp. 977-990, 1996.

[8] G. Zhu, A. Kaddouri, L. A. Dessaint, and O. Akhrif, "A nonlinear state observer for the sensorless control of a permanent-magnet AC machine," IEEE Transactions on Industrial Electronics, vol. 48, no. 6, pp. 1098-1108, 2001.

[9] A. J. Krener, "Approximate linearization by state feedback and coordinate change," Systems and Control Letters, vol. 5, no. 3, pp. 181-185, 1984.

[10] W. Kang and A. J. Krener, "Extended quadratic controller normal form and dynamic state feedback linearization of nonlinear systems," SIAM Journal on Control and Optimization, vol. 30, no. 6, pp. 1319-1337, 1992.

[11] A. J. Krener and W. Kang, "Extended normal forms of quadratic systems," in Proceedings of the 29th IEEE Conference on Decision and Control, pp. 2091-2096, IEEE, New York, NY, USA, December 1990.

[12] V. I. Arnold, Geometric Methods in the Theory of Ordinary Differential Equations, Springer, New York, NY, USA, 1983.

[13] A. U. Levin and K. S. Narendra, "Control of nonlinear dynamical systems using neural networks. Controllability and 
stabilization," IEEE Transactions on Neural Networks, vol. 4, no. 2, pp. 192-206, 1993.

[14] A. K. Parvathy, V. Kamaraj, and R. Devanathan, "A new linearisation technique for permanent magnet synchronous motor model," in Proceedings of the Joint International Conference on Power System Technology and IEEE Power India Conference (POWERCON '08), pp. 1-5, New Delhi, India, October 2008.

[15] A. K. Parvathy, R. Devanathan, and V. Kamaraj, "Application of quadratic linearization to control of Permanent Magnet synchronous motor," in Proceedings of the 1st International Conference on Electrical Energy Systems (ICEES '11), pp. 158-163, 2011.

[16] B. C. Kuo, Automatic Control Systems, Prentice-Hall, New Delhi, India, 2001.

[17] P. Brunovsky, "A classification of linear controllable systems," Kybernetika, vol. 6, no. 3, pp. 173-188, 1970.

[18] G. S. Cardoso and L. Schnitman, "Analysis of exact linearization and aproximate feedback linearization techniques," Mathematical Problems in Engineering, vol. 2011, Article ID 205939, 17 pages, 2011.

[19] A. K. Parvathy, V. Kamaraj, and R. Devanathan, "Complete quadratic linearisation of machine models," in Proceedings of the IEEE International Conference on Control Applications, pp. 1130-1133, Singapore, October 2007. 

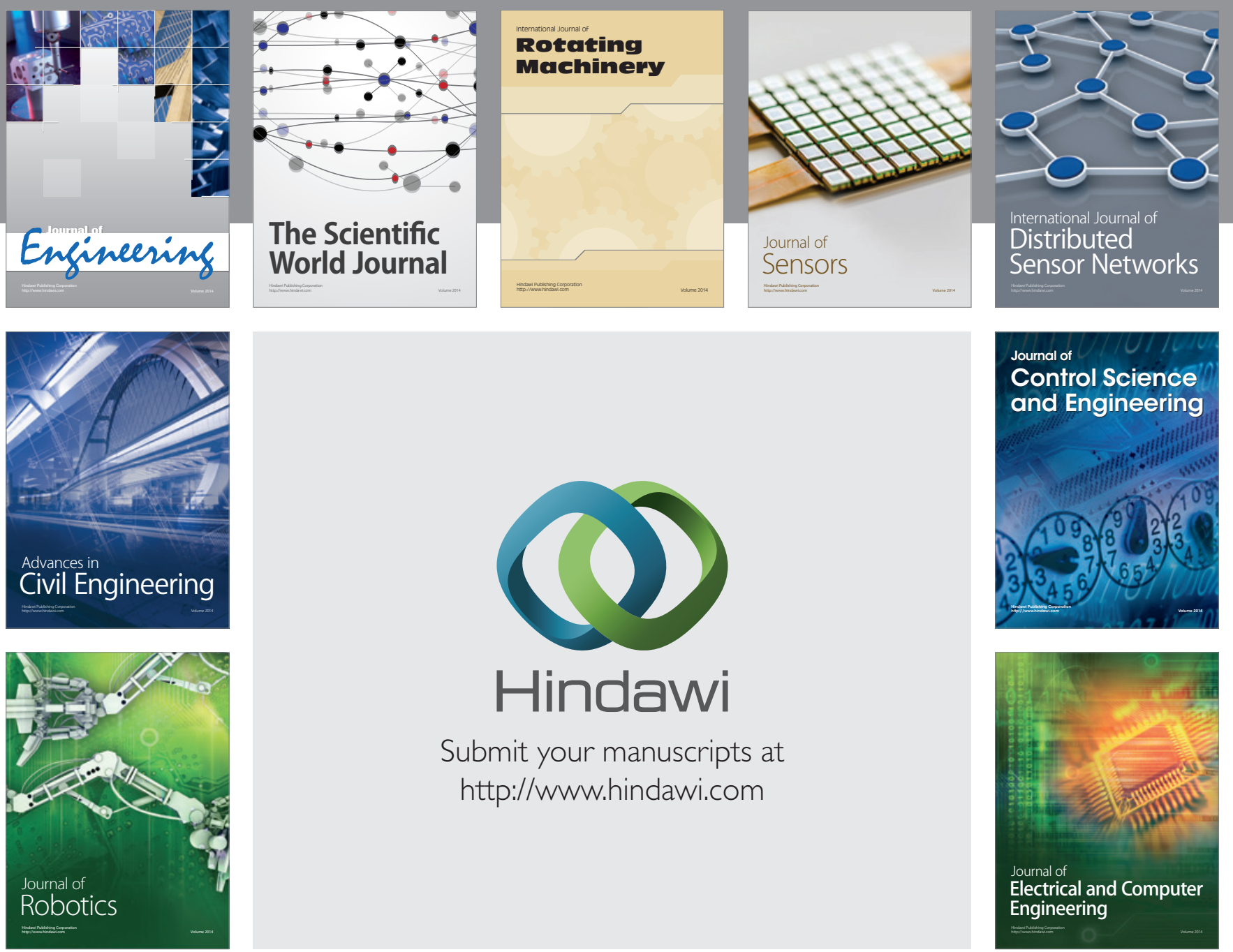

Submit your manuscripts at

http://www.hindawi.com
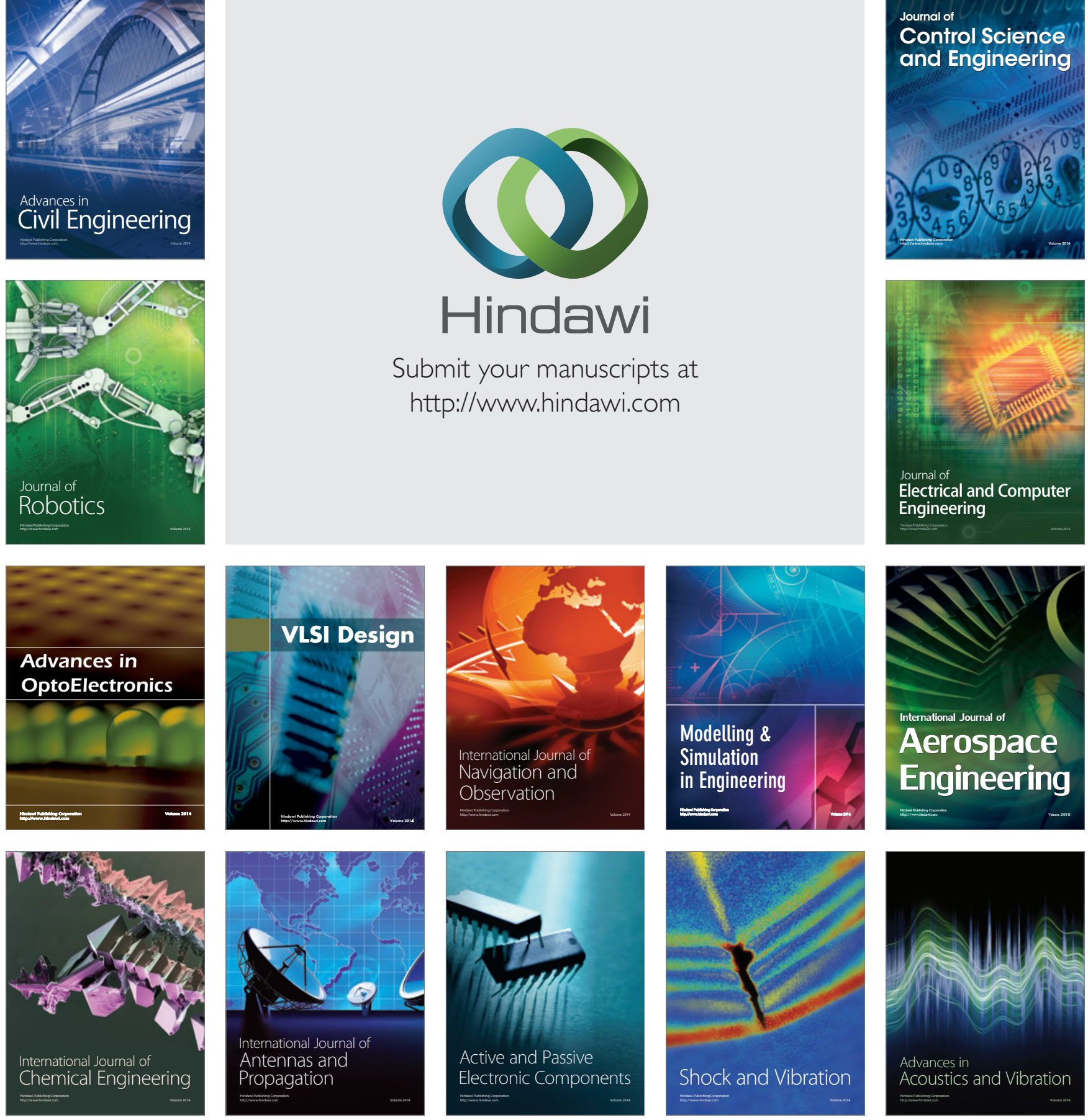